\title{
Sensibilidad a la recompensa, adicción a la comida y obesidad en madres e hijos
}

\author{
Sensitivity to reward, food addiction and obesity in mothers and \\ children
}

\section{Sensibilidade à recompensa, dependência alimentar e obesidade em mães e filhos}

\author{
Jesús Melchor Santos-Flores ${ }^{1}$ \\ (iD) https://orcid.org/0000-0002-9110-9575 \\ Velia Margarita Cárdenas-Villarreal ${ }^{2}$ \\ (iD) https://orcid.org/0000-0001-9315-3193 \\ Juana Mercedes Gutierrez-Valverde ${ }^{3}$ \\ (iD https://orcid.org/0000-0001-9506-5947 \\ Luis Arturo Pacheco-Pérez ${ }^{4}$ \\ (iD) https://orcid.org/0000-0002-3765-5559 \\ María de los Ángeles Paz- Morales ${ }^{5}$ \\ (iD https://orcid.org/0000-0002-4111-8499 \\ Milton Carlos Guevara-Valtier ${ }^{6 *}$ \\ (iD) https://orcid.org/0000-0001-7291-3931
}

1. Maestría en Ciencias de Enfermería. Universidad Autónoma de Nuevo León., Facultad de Enfermería. Monterrey, N. L., México.

2. Doctorado en Cultura del Cuidado de Enfermería. Universidad Autónoma de Nuevo León, Facultad de Enfermería. Monterrey, N. L., México.

3. Doctorado en Ciencias de Enfermería. Universidad Autónoma de Nuevo León, Facultad de Enfermería. Monterrey, N. L.., México.

4. Doctorado en Ciencias de Enfermería. Universidad Autónoma de Nuevo León, Facultad de Enfermería. Monterrey, N. L., México.

5. Doctorado en Educación. Universidad Autónoma de Nuevo León, Facultad de Enfermería.

6. Doctorado en Educación. Universidad Autónoma de Nuevo León, Facultad de Enfermería

*Autor para correspondencia: milton.guevarav@uanl.mx

Recibido: $21 / 02 / 2021$

Aceptado: $11 / 08 / 2021$ 


\title{
Resumen
}

Introducción: El sobrepeso y la obesidad se consideran una epidemia que afecta a la población de todas las edades. Las características presentes en las diada madre e hijo escolar como la sensibilidad a la recompensa al comer en exceso y la adicción a la comida pueden ser potenciales reguladores del peso corporal. Objetivo: Conocer la influencia de la sensibilidad a la recompensa, la adicción a la comida y estado nutricio materno sobre la sensibilidad a la recompensa, la adicción a la comida y estado nutricio del hijo. Metodología: Diseño descriptivo-correlacional. Muestra conformada por 218 diada. Se midió el peso y talla, se aplicó una cédula de datos sociodemográficos, y cuatro instrumentos de medición. Los datos se capturaron y analizaron mediante el SPSS. Resultados: El 69.3\% de las madres presentaron sobrepeso y obesidad, en los hijos el 27.9\%. Reportaron alta sensibilidad a la recompensa (38.4 y 40.4), medias mayores en aquellos con sobrepeso y obesidad $(p<.001)$. El $13.8 \%$ de las madres y el $8.3 \%$ de los hijos presentaron adicción a la comida. Madres e hijos con adicción a la comida presentaron mayor puntaje de sensibilidad a la recompensa $(p<.001)$. El índice de masa corporal, sensibilidad a la recompensa y adicción a la comida de la madre influyeron sobre el índice de masa corporal del hijo $(p<.005)$. Conclusiones: Estas características de la madre estarían presentes en los niños, podrían aumentar conforme aumenta la edad, persistir en la edad adulta y ser trasmitidas hacia sus futuros hijos.

Palabras clave: Obesidad; Adicción a la Comida; Recompensa; Conducta Materna (DeCS).

\begin{abstract}
Introduction: Overweight and obesity are considered an epidemic that affects the population of all ages. The characteristics present in the mother and school age children dyads such as sensitivity to the reward upon eating in excess and the addiction to food can be potential regulators of body weight. Objective: Understand the influence of sensitivity to rewards, addiction to food and the maternal nutritional status in regard to sensitivity to rewards, addiction to food and nutritional status of children. Methodology: Descriptive-correlational design. Sample composed by 218 pairs. Weight and size were measured, applying a sociodemographic data card, as well as four measurement instruments. The data was captured and analyzed throughout SPSS. Results: $69.3 \%$ of the mothers were overweight and obese, and $27.9 \%$ of the children. They reported high sensitivity to reward (38.4 and 40.4), and mean higher in those overweight and obese $(p<.001$ ). $13.8 \%$ of mothers and $8.3 \%$ of the children presented addiction to food. Mothers and children with addiction to food showed a higher score of sensitivity to reward $(p<.001)$. The body mass index, sensitivity to reward and addiction to food on the mothers' behalf influenced the body mass index of the child $(p<.005)$. Conclusions: These maternal characteristics would be present in children, they can increase according to age, persist into adult age and be transmitted towards their future children.
\end{abstract}

Key words: Obesity; Food Addiction; Reward; Maternal Behavior (DeCS). 


\begin{abstract}
Abstrato
Introdução: O sobrepeso e a obesidades ao considerados uma epidemia que afeta a população de todas as idades. As características presentes nas díades da mãe e do filho na escola, como a sensibilidade à recompensa por comer demais e dependência alimentar, podem ser potenciais reguladores do peso corporal. Objetivo: Conhecer a influência da sensibilidade à recompensa, o vício no alimento e o estado nutricional materno na sensibilidade e à recompensa, o vício no alimento e o estado nutricional da criança. Metodologia: Desenho descritivo-correlacional. Amostra composta por 218 díades. O peso e a estatura foram medidos, um cartão de dados socio-demographic foi aplicado e quatro instrumentos de medição. Os dados foram capturados e analisados usando o SPSS. Resultados: O 69,3\% das mães presentavam sobrepeso e obesidade, nas crianças $27,9 \%$. Eles relatara malta sensibilidade à recompensa $(38,4$ e 40,4$)$, médias mais altas naqueles com sobrepeso e obesidade ( $p<0,001)$. 13,8\% das mães e $8,3 \%$ das filhos tinham dependência alimentar. Mães e crianças com dependência alimentar tivera um maior e score de sensibilidade à recompensa $(p<0,001)$. O índice de massa corporal, a sensibilidade à recompensa e a dependência alimentar da mãe influenciaram o índice de massa corporal da criança $(p<0,005)$. Conclusões: Essas características da mãe estariam presentes nos filhos, poderiam aumentar com o aumento da idade, persistir na ida de adulta e serem transmitidas a os futuros filhos.
\end{abstract}

Palavras-chave: Obesidade; Dependência alimentar; Recompensa; Comportamento materno (DeCS).

\title{
Introducción
}

El sobrepeso y la obesidad (SP/OB) se consideran una epidemia ${ }^{(1,2)}$ y el quinto factor de riesgo de muerte a nivel mundial, son consideradas como primeras causas de diabetes mellitus tipo 2 y de otras enfermedades como cardiopatías isquémicas, algunos tipos de cáncer y muerte prematura (3). La Organización Mundial de la Salud ${ }^{(4)}$ reporta que $52 \%$ de la población mundial adulta presenta SP/OB y $18 \%$ de la población infantil. En México las prevalencias son mayores un $72 \%$ en población adulta y $33.2 \%$ en niños presentan esta condición ${ }^{(5)}$.

El aumento del SP/OB tanto en adultos como en niños hace necesario conocer las causas que lo generan. Estudios han identificado factores contribuyentes al SP/OB, incluyendo el acceso a la comida hipercalórica, el consumo de cantidades de comida desmesurada y la reducción del ejercicio ${ }^{(6,7)}$. Sin embargo, recientemente algunas variables que han cobrado interés para explicar el desarrollo del SP/OB 
han sido la sensibilidad a la recompensa (SR) al comer en exceso y la adicción a la comida (AC), los cuales pueden ser considerados como posibles reguladores del peso corporal ${ }^{(7,8)}$.

La SR se refiere a la búsqueda de sustancias, experiencias apetitivas y gratificantes en el contexto de la alimentación ${ }^{(9)}$. Se ha observado en madres de familia con alta SR algunos problemas alimenticios asociados con el SP/OB así mismo la $\mathrm{AC}$ refiriéndose a esta como a la ingesta excesiva de alimentos con alto contenido calórico acompañada de pérdida de control y antojos intensos de alimentos, algunos autores mencionan que podría ser un fenotipo del SP/OB $(6,8,10,11-14)$.

Tanto la SR y la AC podrían estar relacionadas con el SP/OB, existe la posibilidad directamente proporcional de que los niños con alta SR y AC tengan madres con esa misma situación ${ }^{(15)}$. Dado que las preferencias y rechazos hacia determinados alimentos en los hijos escolares pudieran estar condicionados principalmente por la madre, considerada la principal proveedora y responsable de preparar alimentos en el hogar en la mayoría de los casos $(9,15,16)$, es importante conocer la relación de estas variables puesto que el grupo de menores de edad se encuentra en riesgo de desarrollar la SR asociada con una conducta adictiva y con el SP/OB influenciado principalmente por conductas maternas $(7,9,14,15,17,18)$.

El presente estudio contribuirá al repertorio de conocimientos de enfermería y de la salud en general a identificar factores de la conducta alimentaria y comprender las conductas adictivas de recompensa y de qué manera esta se ve influenciada por la madre en el hijo ${ }^{(15,17)}$, se podrán desarrollar estrategias de prevención y promoción eficaces enfocadas en la disminución del impacto de las conductas maternas en el hijo ${ }^{(9)}$. Por lo anterior, el propósito del presente estudio fue determinar la influencia de la sensibilidad a la recompensa, adicción a la comida, y estado nutricional de la madre sobre la sensibilidad a la recompensa, adicción a la comida y estado nutricional del hijo escolar. 


\section{Hipótesis de investigación}

Ho= El índice de masa corporal, la sensibilidad a la recompensa y la adicción a la comida de la madre no influyen sobre el índice de masa corporal, la sensibilidad a la recompensa y la adicción a la comida en el hijo escolar.

$\mathrm{Ha}=\mathrm{El}$ índice de masa corporal, la sensibilidad a la recompensa y la adicción a la comida de la madre influyen sobre el índice de masa corporal, la sensibilidad a la recompensa y la adicción a la comida en el hijo escolar.

\section{Metodología}

\section{Diseño del estudio}

El presente estudio fue de tipo descriptivo-correlacional.

\section{Variables de estudio}

La SR materna es la conducta manifestada por la madre sobre la búsqueda de alimentos que le desencadenan experiencias apetitivas y gratificantes, sentirse emocionada, llena de energía y tener ganas de experimentar nuevas sensaciones. La SR del hijo es la conducta del hijo observada por la madre sobre los alimentos que le desencadenan experiencias apetitivas y gratificantes, lo hacen sentir emocionado, lleno de energía y consumir alimentos por gusto anhelando nuevas emociones y sensaciones. La AC materna es la conducta que manifiesta la madre sobre la ingesta excesiva de alimentos con alto contenido calórico acompañada de pérdida de control, tolerancia, abstinencia, deseo, mayor tiempo invertido en el consumo de alimentos, abandono de actividades importantes, consumo a pesar de las consecuencias y una alteración clínica importante. La AC del hijo escolar, es la conducta que manifiesta el hijo escolar sobre la ingesta excesiva de alimentos con alto contenido calórico acompañada de pérdida de control, tolerancia, abstinencia, deseo, mayor tiempo invertido en el consumo de alimentos, abandono de actividades importantes, consumo a pesar de las consecuencias y una alteración clínica importante. 
El índice de masa corporal (IMC) en la madre es el resultado de dividir el peso en kilogramos por la talla en metros al cuadrado $\left(\mathrm{kg} / \mathrm{m}^{2}\right)$, de bajo peso $(<18.5)$, peso normal (18.5-24.9), sobrepeso (25-29.9), obesidad (>30). El IMC en el hijo escolar es el resultado de dividir el peso en kilogramos por la talla en metros al cuadrado $\left(\mathrm{kg} / \mathrm{m}^{2}\right)$, considerando el percentil para peso normal $(<85)$, sobrepeso (85-94.9) y obesidad $(\geq 95)$ dependiendo de la edad y sexo. La edad de la madre fue el tiempo que ha transcurrido desde el nacimiento de la madre, se evaluó en términos de años que reportó tener la madre al momento de la entrevista, así como la edad del hijo escolar fue el tiempo que ha transcurrido desde el nacimiento del escolar, se evaluó en términos de años que reportó tener el hijo escolar al momento de la entrevista y el sexo del hijo escolar es lo que se atribuye a las mujeres y a los hombres social y culturalmente a partir del sexo biológico, se evaluó correspondientemente al reporte verbal en términos de femenino o masculino que reporte el hijo escolar.

\section{Población, muestra y muestreo}

La población de interés estuvo conformada por 703 diadas (madres e hijos de entre 6 a 12 años), escolarizados en una institución de educación pública de Nuevo León. El muestreo fue probabilístico por conglomerados unietápico.

Para determinar el tamaño de la muestra se utilizó el paquete estadístico nQuary Advisor ${ }^{\circledR}$ versión V7.0, se estimó a partir de un Modelo de Regresión Lineal Múltiple con 5 variables independientes con un nivel de significancia de .05 , una potencia del .90 y un Coeficiente de Determinación $R^{2}$ de $.08{ }^{(19)}$. Se obtuvo una muestra de 196 participantes, al considerar una tasa de no respuesta del 10\% se obtuvieron 218 diada madre e hijo escolar.

\section{Criterios de inclusión}

Madres y niños escolares que puedan permanecer de pie al menos 5 minutos para la medición de peso y talla. Madres de niños escolares que acepten su participación, así como la de su hijo de forma voluntaria en el estudio. 


\section{Criterios de exclusión}

Niños escolares con alguna incapacidad física. Madres con analfabetismo y/o alguna limitación física.

\section{Mediciones}

Se aplicó una cédula de datos sociodemográficos para registrar la edad (años), sexo (masculino y femenino), escolaridad (años de estudio), peso $(\mathrm{kg})$ y talla $(\mathrm{cm})$ y cuatro cuestionarios. Para medir la SR materna se utilizó la Escala del Sistema de Activación Conductual (BAS, por sus siglas en inglés) de Carver (20) en su versión al español (21). La SR del hijo escolar mediante la Escala del Sistema de Activación Conductual versión para padres (BASP, por sus siglas en inglés) (22).

La escala BAS y BASP tienen 13 ítems respectivamente, se dividen en 3 subescalas, la primera es Sensibilidad a la Recompensa con 5 ítems (2, 4, 8, 10 y 13), la segunda se denomina Motivación con 4 ítems (1, 5, 7

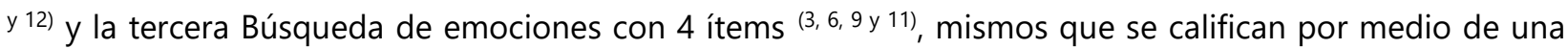
escala Likert de 4 puntos que van desde $1=$ Totalmente en desacuerdo a $4=$ Totalmente de acuerdo, las puntuaciones más altas indican una mayor SR. La escala BAS obtuvo un $\alpha$ de Cronbach de .93 en el presente estudio, mientras que la escala BASP un $\alpha$ de Cronbach de .94.

Con la Escala de Adicción a la Comida de Yale (YFAS) ${ }^{(23)}$ se midió la AC de la madre, con respecto a la AC del hijo esta se midió con la Escala de Adicción a la Comida de Yale versión para niños (YFAS-C) (15). Están basadas en los criterios de dependencia de sustancias del Diagnostic Medical Sonography (DMS-V) al ajuste del comportamiento alimentario. Estas medidas de autoinforme constan de 25 ítems, incluyen respuestas tipo Likert (nunca hasta 4 o más veces a la semana) y dicotómicas (sí o no). Las escalas constan de 7 dimensiones de síntomas, que se distribuyen de la siguiente manera: 1) mucho tiempo invertido en el consumo, 2) tolerancia, 3) abstinencia, 4) deseo persistente, 5) alimento consumido en mayor cantidad y durante un período más largo de tiempo, 6) abandono de actividades importantes, 7) consumo a pesar de las consecuencias, y significancia clínica. 
El diagnóstico de la AC, se obtiene a través de la suma de al menos 3 síntomas y 1 significancia clínica. En el presente estudio la escala YFAS presentó un $\alpha$ de Cronbach de .94. Mientras que la escala YFASC un $\alpha$ de Cronbach de .92.

Para evaluar el estado nutricional, se llevaron a cabo mediciones de peso y talla mediante una báscula marca SECA 750 previamente calibrada se realizó la medición de peso y mediante un estadímetro portátil marca SECA 213 la toma de talla tanto para la madre como para el hijo. Se obtuvo, el Índice de Masa Corporal (IMC) mediante la fórmula IMC=kg/ $\mathrm{m}^{2}$, con éste, se clasificó a cada madre de acuerdo a criterios de la OMS (24) en condición de bajo peso (<18.5), peso normal (18.5-24.9), sobrepeso (25-29.9) y obesidad (>30), para el hijo se utilizó la clasificación de la CDC ${ }^{(25)}$ considerando el percentil para peso normal $(<85)$, sobrepeso (85-94.9) y obesidad ( $\geq 95)$ dependiendo de la edad y sexo.

\section{Procedimientos}

En primera instancia se solicitaron las autorizaciones de los Comités de Investigación y Ética en Investigación de la Facultad de Enfermería de la Universidad Autónoma de Nuevo León y de las autoridades de la institución educativa. El número de registro del comité fue F-1503.

Se entregó una invitación a los niños para citar a sus madres y así mismo se les brindó el consentimiento informado para madres y la carta de asentimiento para niños días previos a la administración de los instrumentos de medición.

Una vez que las madres enviaron de vuelta la invitación a través de sus hijos se revisaron los consentimientos informados y asentimientos de las madres que aceptaron formar parte del estudio. En los días y horarios programados se les pidió que se ubicaran en un aula condicionada, nuevamente se explicó el objetivo y las instrucciones para contestar cada instrumento protegiendo la privacidad tanto de la madre como del hijo mediante el anonimato. Posteriormente se procedió a la medición del peso y talla conforme a las consideraciones determinadas en la NOM-031-SSA2-1999, NOM-043-SSA2-2012 y NOM-047-SSA22015. 


\section{Análisis estadístico}

Los datos se capturaron y analizaron mediante el uso del paquete estadístico Statistical Package for the Social Sciences (SPSS) versión 22 para Windows. Se utilizó estadística descriptiva para variables sociodemográficas e inferencial para variables de estudio. Se utilizó U de Mann-Whitney, Chi-cuadrado de Pearson y el Coeficiente de Correlación de Spearman. Para la estimación de los efectos sobre variables dependientes continuas, como el IMC y la SR, se recurrió a Modelos de Regresión Lineal Múltiple y Regresión Logística en el caso de la AC.

\section{Consideraciones éticas}

El estudio se apegó a lo dispuesto en el reglamento de la Ley General de Salud en Materia de Investigación para la Salud (26). El presente estudio se apegó a los aspectos éticos para el desarrollo de la investigación en seres humanos.

\section{Resultados}

Participaron 218 diadas. El 57.3\% de las madres se encontraban casadas y el $71.6 \%$ se dedicaban al hogar, la media de edad fue de 33.95 años $(D E= \pm 4.86)$, el promedio de años de estudio fue de $11.9(D E= \pm 2.45)$. Con respecto a las características antropométricas el 38.1\% presentó SP y el 31.7\% OB, la media de IMC fue de $27.8(D E= \pm 4.83)$.

De acuerdo a los datos sociodemográficos y antropométricos de los hijos escolares, se aprecia que el $50.9 \%$ de las participantes fueron de sexo femenino, la media de edad fue de 9.8 años $(D E= \pm 1.49)$. Con respecto a las características antropométricas el $72 \%$ de los hijos se encontraron en el percentil de IMC de peso normal, el $12.80 \%$ en SP y el $15.1 \%$ en OB. La media de IMC fue de $18.1(D E= \pm 3.95)$.

De acuerdo a la SR materna y del hijo escolar; se presentó una media de SR de $38.4(D E= \pm 10.78)$ en madres. Con respecto a la comparación entre madres con IMC normal la media de SR fue de 29.44 ( $D E$ $= \pm 12.12)$, siendo mayor en madres con SP $(\bar{X}=41.2, D E= \pm 7.89)$ y $\mathrm{OB}(\bar{X}=43.7, D E= \pm 6.83)$. Las madres 
con un IMC más elevado presentaron medias de puntajes de SR más altas $(H=49.64, p<.001)$. No se presentaron diferencias significativas en cuanto a la edad $(p>.05)$.

En hijos se reportó una media de SR de $40.4(D E= \pm 10.50)$, con respecto a la comparación entre hijos con IMC normal la media de SR fue de $38.7(D E= \pm 11.21)$, siendo mayor en hijos con SP $(\bar{X}=42.4, D E= \pm 7.98)$ y OB $(\bar{X}=47.1, D E= \pm 5.59)$, los hijos con IMC elevado presentaron una SR más alta en comparación con los hijos de IMC normal $(H=20.73, p<.001)$. No se reportaron diferencias significativas en cuanto a la edad y sexo $(p>.05)$.

Con respecto a la AC, el $13.8 \%$ de las madres y el $8.3 \%$ de los hijos presentaron AC. De acuerdo a la categoría de IMC se reportó en mayor frecuencia la AC en madres con SP (10.8\%) y OB (29\%), en madres con IMC normal se presentó la AC en menor frecuencia (1.50\%). Se reportó un mayor porcentaje de AC en hijos con edades de entre 11 y 13 años (16.5\%), en comparación con hijos más jóvenes quienes reportaron porcentajes de entre 2.5 y 3.8\% $\left(X^{2}=12.477, p=.002\right)$. De acuerdo a la categoría de IMC se reportó en mayor frecuencia la AC en hijos con SP (14.3\%) y OB (24.2\%), en hijos con IMC normal se presentó en menor frecuencia (3.8\%) $\left(X^{2}=16.553, p<.001\right)$, estas diferencias fueron significativas.

En la tabla 1, las madres con AC presentaron una media de SR mayor en comparación con las madres $\sin A C(U=1363, p=.001)$. Así mismo los hijos con madres que presentaron AC, tenían una media de SR mayor $(U=1375.50, p=.001)$. De acuerdo a las madres con hijos que presentaron AC, estas presentaron una media de SR más alta en comparación con aquellas madres que tuvieron hijos $\sin A C(U=$ $664, p=.001)$. Con respecto a los hijos con $A C$, tuvieron una SR más alta que aquellos $\sin A C(U=681.50, p$ $=.001)$.

Las variables SR materna y el IMC de la madre se correlacionaron de manera positiva y significativa $(r s=.442, p=.001)$, a mayor SR materna mayor era el IMC. En el caso de las variables de SR del hijo y el IMC del hijo de igual manera se encontró correlación positiva y significativa ( $r s=.326, p<.001)$, a mayor SR del hijo mayor era su IMC. Se encontró correlación positiva y significativa entre la SR materna y la SR del 
hijo $(r s=.837, p<.001)$, es decir, a mayor SR en la madre mayor era la SR en el hijo. Entre el IMC de la madre y el IMC del hijo ( $r s=.461, p<.001)$, a mayor IMC de la madre mayor era el IMC en su hijo.

Tabla 1. Diferencia en la AC de acuerdo a SR en madres e hijos

\begin{tabular}{|c|c|c|c|c|c|c|c|c|}
\hline \multirow{2}{*}{\multicolumn{2}{|c|}{ AC de la madre }} & \multirow{2}{*}{$n$} & \multicolumn{5}{|c|}{$S R$} & \multirow{2}{*}{$U$} \\
\hline & & & $\bar{X}$ & $M d n$ & $D E$ & Valor Min & Valor Max & \\
\hline \multirow{2}{*}{ Madre } & Con AC & 30 & 46.00 & 48.50 & 7.40 & 28 & 52 & \multirow{2}{*}{$1363^{* *}$} \\
\hline & $\operatorname{Sin} A C$ & 188 & 37.24 & 40.00 & 10.76 & 13 & 52 & \\
\hline \multirow{2}{*}{ Hijo } & Con AC & 30 & 47.00 & 50.00 & 7.55 & 19 & 52 & \multirow{2}{*}{$1375.50^{\star \star}$} \\
\hline & $\operatorname{Sin} A C$ & 188 & 39.43 & 43.00 & 10.65 & 13 & 52 & \\
\hline & \multicolumn{8}{|c|}{$S R$} \\
\hline \multicolumn{2}{|c|}{ AC del hijo } & $n$ & $\bar{X}$ & Mdn & $D E$ & Valor Min & Valor Max & \\
\hline \multirow{2}{*}{ Madre } & Con AC & 18 & 47.67 & 52.00 & 7.68 & 28 & 52 & \multirow{2}{*}{$664.0^{* *}$} \\
\hline & $\operatorname{Sin} A C$ & 200 & 37.62 & 40.50 & 10.64 & 13 & 52 & \\
\hline \multirow{2}{*}{ Hijo } & Con AC & 18 & 48.44 & 52.00 & 6.55 & 32 & 52 & \multirow{2}{*}{$681.5^{\star *}$} \\
\hline & $\operatorname{Sin} A C$ & 200 & 39.76 & 43.00 & 10.61 & 13 & 52 & \\
\hline
\end{tabular}

Nota: $\bar{X}=$ Media, Mdn = mediana, $D E=$ desviación estándar, Valor Min = valor mínimo, Valor Max = valor máximo, $U=U$ de Mann-Whitney, ${ }^{* *}=p<.001, n=218$

Se realizó un Modelo de Regresión Lineal Múltiple con el método "Backward" con la SR del hijo como variable dependiente; se encontró que la SR materna presentó significancia estadística $(p=.001)$, explicó un $75.8 \%$ de la varianza, $F(1,217)=680.52, p=.001$, IC 95\% [4.96, 10.13] (Figura 1).

Figura 1. Efecto de la SR materna sobre la SR del hijo.

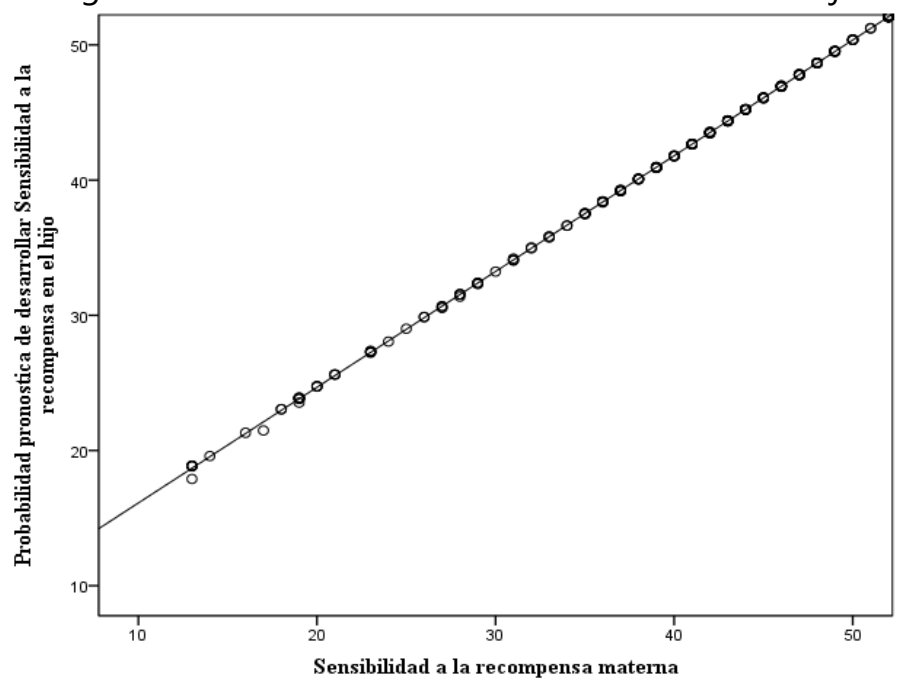

Fuente: Elaboración propia 
Posteriormente se realizó un Modelo de Regresión Logística con el método "Backward" con la AC del hijo como variable dependiente. Las variables que contribuyen al modelo son la SR materna $(O R=1.122$, $p=.019)$ y la $A C$ de la madre $(\mathrm{OR}=9.381, p=.001)$ observando significancia estadística $\left(\mathrm{R}^{2}=37.6 \%, p=\right.$ .001) (Figura 2).

Figura 2. Efecto de la SR y AC materna sobre la AC del hijo.

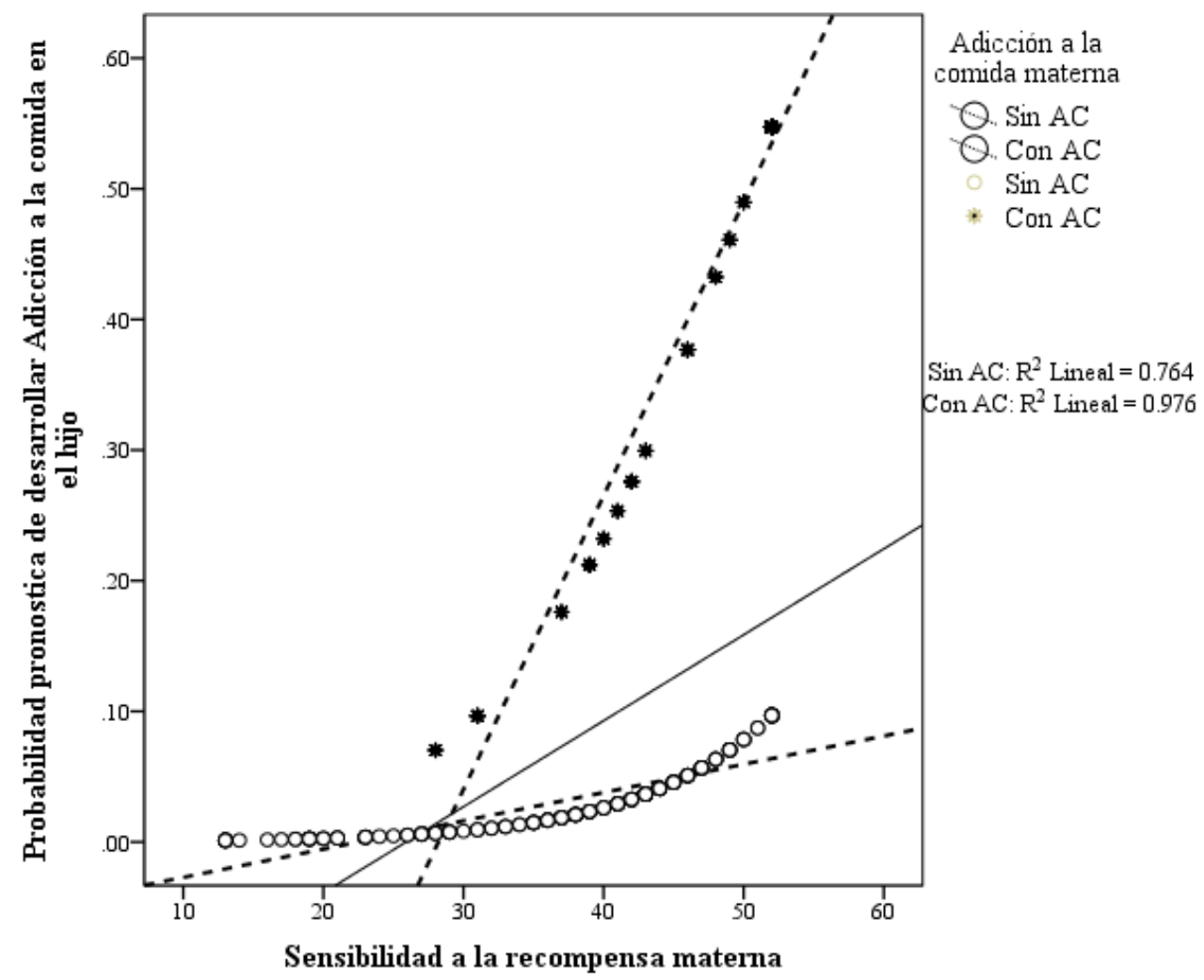

Fuente: Elaboración propia

En cuanto a las variables que influyeron en el IMC del hijo. Los resultados de los Coeficientes de Regresión Lineal Múltiple muestran solo un modelo con significancia estadística, $F(5,212)=25.92, p=.001$, IC 95\% $[5.75,11.44]$. La SR materna, AC de la madre e IMC de la madre influyen de manera significativa sobre el IMC del hijo ( $p=.001)$ (Figura 3 y 4$)$. 
Figura 3. Efecto de la SR e IMC de la madre sobre el IMC del hijo.

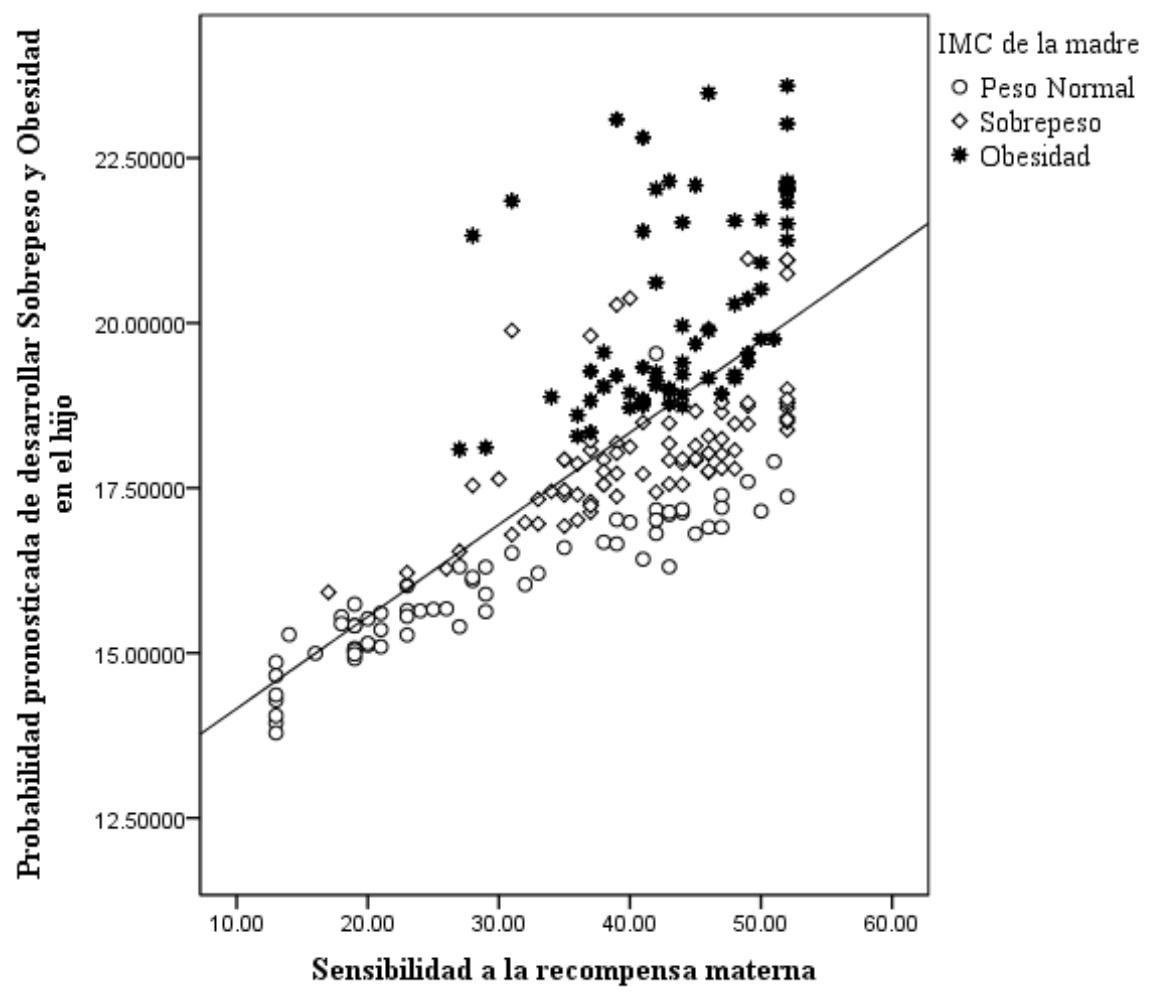

Fuente: Elaboración propia

Figura 4. Efecto de la SR y AC materna sobre el IMC del hijo.

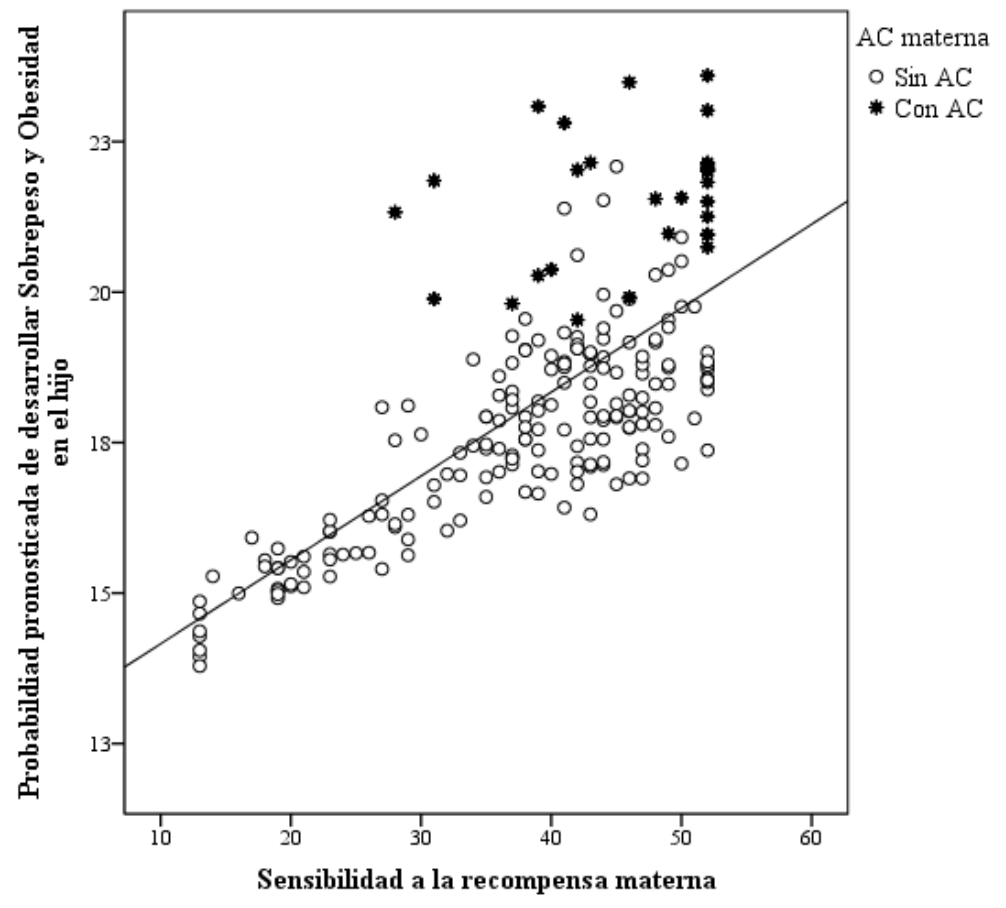

Fuente: Elaboración propia 


\section{Discusión}

El presente estudio se incorpora a la literatura limitada sobre la SR y la AC en diada madre e hijo escolar, basada en el autoinforme de la madre y del hijo. Los resultados en gran parte replicaron los hallazgos de otros estudios realizados en adultos y niños y demuestra que el BAS, BASP, YFAS y YFAS-C son medidas apropiadas para evaluar la SR y AC en ambas poblaciones, de este modo se puede determinar que la SR y la AC no están restringidas a la población adulta, sino que también se presentan a temprana edad en niños escolares $(8,9,15,27)$.

En cuanto a las características antropométricas en las madres, la mayoría presentaron sobrepeso y obesidad (SP/OB), se reportó una media de Índice de Masa Corporal (IMC) de $27.28 \mathrm{~kg} / \mathrm{m}^{2}$, similar a lo encontrado por diferentes autores ${ }^{(28)}$ donde reportaron una media de $27.40 \mathrm{~kg} / \mathrm{m}^{2}$, esta cifra sigue siendo superior a lo encontrado por la mayoría de las investigaciones donde la media de IMC de los adultos osciló entre 21.70 y $26.90 \mathrm{~kg} / \mathrm{m}^{2}(8,13,14,15)$. En el contexto mexicano las prevalencias de SP/OB son elevadas y unas de las más altas a nivel mundial, siendo superiores a las prevalencias de los países donde realizaron los estudios previos, en México el SP/OB se encuentra presente en un $72 \%$ de la población adulta, los resultados del presente estudio son similares a los resultados a nivel nacional ${ }^{(5)}$.

En los hijos, 28\% fueron identificados con SP/OB, similar a lo reportado por otros autores ${ }^{(18,29)}$ y coincidiendo con los reportes emitidos por la Encuesta Nacional de Salud y Nutrición ${ }^{(5)}$ donde señala que 33.2\% de la población infantil presenta esta condición de salud a nivel nacional.

La media de SR en madres (38.44) fue superior a lo reportado por otros estudios $(8,13,14)$. Dado que las madres con SP/OB presentaron medias más altas de SR posiblemente las diferencias en cuanto a los puntajes de SR pudieran deberse a la alta prevalencia de SP/OB reportada en el estudio actual, quizá las madres con SP/OB tengan densidades de receptores dopaminérgicos D2 más bajas, lo que implica una alta SR, además es probable que presenten neuroadaptaciones a la hiperactividad de los circuitos de recompensa a nivel cerebral, sirviendo como un factor de riesgo para comer en exceso y tener SP/OB ${ }^{(30)}$. 
La media de SR en los hijos (40.47) fue más alta que la reportada por otros autores en donde la media obtenida fue entre 32.10 a $34.57^{(9,17,18)}$. La SR no presentó diferencias significativas según sexo y edad de los hijos, este hallazgo concuerda con lo reportado por estudios previos $(9,17,18)$. En cuanto a la SR y el IMC, los hijos con alta SR presentaron mayor SP/OB, la mayor parte de la literatura ha reportado asociaciones positivas entre la SR y el IMC en niños con SP/OB $(9,14,31,32)$. Así mismo, se encontró relación positiva y significativa entre la SR del hijo y su IMC, similar a los resultados de los estudios previos $O B(9,14,31,32)$.

El hallazgo del presente estudio concuerda con la premisa de que los niños con SP/OB tienen más probabilidades de buscar y disfrutar de las recompensas en cuanto a la comida. En consecuencia, estos niños probablemente comerán en exceso cuando los alimentos sabrosos, hipercalóricos y altos en azúcar estén disponibles ${ }^{(9)}$. Se encontró relación positiva y significativa entre la SR materna y la SR del hijo, a diferencia de las madres que toman sus propias decisiones en cuanto a la selección de alimentos y horarios de comida, en los niños a menudo estas decisiones son dictadas por sus madres ${ }^{(15)}$.

Algunos estudios han encontrado que la exposición frecuente, por parte de la madre hacia su hijo, a los alimentos hipercalóricos, altos en grasas y azúcares produce una alteración en la SR, lo que lleva a un aumento en la ingesta de alimentos hipercalóricos ${ }^{(33)}$. Existe la posibilidad de que la SR materna conduzca a una alteración desde un nivel genético y, por lo tanto, afectar el control del apetito y las preferencia por el consumo de alimentos inapropiados en sus hijos ${ }^{(33,34)}$, quizá la alta SR y el SP/OB de los hijos estén siendo programados desde el momento de la concepción ${ }^{(35)}$ y las vías de recompensa se desarrollen en el útero alterando las respuestas neuroadaptativas de la SR en el hijo ${ }^{(33)}$.

Respecto a la AC, el 13.8\% de las madres presentaron esta condición. Se presentó una mayor prevalencia de $\mathrm{AC}$ en madres mayores de 40 años, estas proporciones no presentaron diferencias significativas, similar a la mayoría de los estudios previos ${ }^{(10,13,15,28)}$. En cuanto a la AC y el IMC en madres, la mayoría de los estudios señalan que existiría mayor prevalencia de AC en individuos con SP/OB (10, 13, 15, 
28), concordante con los hallazgos del presente estudio donde se reportó mayor prevalencia de AC en madres que presentaban SP/OB.

La literatura señala que entre el 56 y el $88.6 \%$ de los adultos con SP/OB presentan AC, lo que proporciona una fuerte evidencia de que la AC puede contribuir a la creciente prevalencia del SP/OB en la población a nivel mundial, los hallazgos actuales sugieren que el SP/OB que se presenta con la AC puede representar un subgrupo importante de individuos con esta condición de salud con una etiología distintiva (28).

El porcentaje de madres con AC del presente estudio (13.8\%) fue superior a lo reportado por otros estudios donde encontraron prevalencias de 5.7 a $12 \%(15,28,36,37)$ e inferior que otros estudios donde encontraron prevalencias de 17.6 a $25 \%$ en mujeres adultas $(10,13,38)$. Estas diferencias pueden ser multicausales, en cuanto a los estudios previos donde las prevalencias de AC fueron menores pudieron deberse a las bajas prevalencias de SP/OB, por otro lado, las diferencias en cuanto a los estudios previos con prevalencias de AC más altas pudieran deberse a los criterios de inclusión y a las técnicas en cuanto a la aplicación del YFAS ${ }^{(15,27)}$.

El porcentaje de hijos con AC del presente estudio (8.3\%) fue inferior a las prevalencias reportadas por las evidencias previas (rango $8.9 \%$ a $41 \%)(29,39,40)$. Las diferencias encontradas en este estudio que constatan una menor prevalencia de AC en relación a otros estudios pudiera ser explicada por las versiones parentales versus el autoinforme de los niños del YFAS-C, las madres que informaron la AC de sus hijos en los estudios previos pudieran subestimar los comportamientos alimenticios de sus hijos y considerarlos problemáticos, siendo más probable que respalden el criterio diagnóstico de AC en sus hijos.

De acuerdo a la AC e IMC en hijos, se presentó diferencia significativa, se reportó un mayor porcentaje de AC en hijos mayores de 11 años, hallazgos similares a lo reportado por otros autores ${ }^{(29)}$, la mayoría de los estudios no encontraron diferencias significativas $(15,39,40)$. Es probable que la AC se presente en edades más tardías ${ }^{(29)}$. 
La mayoría de los estudios señalan que existiría mayor prevalencia de AC en niños con SP/OB (15, 29, 40) hallazgos similares en el presente estudio donde se reportó mayor prevalencia de AC en hijos que presentaban SP/OB.

Las madres e hijos con AC presentaron medias de puntaje de SR más, la asociación entre la SR y la $\mathrm{AC}$ concuerda con otros estudios que muestran una asociación entre la $\mathrm{AC}$ y un perfil genético vinculado a la respuesta a la recompensa en el consumo de alimentos gratificantes ${ }^{(6,13)}$; otros autores que tratan de explicar la alimentación adictiva encuentran que la exposición a alimentos altamente sabrosos podría conducir a adaptaciones neuronales relacionados con la SR ${ }^{(15)}$.

En la adicción relacionada con las drogas, se ha demostrado que el estriado ventral y el mesencéfalo se asocian con recompensas inmediatas y que el hipocampo responde a las consecuencias de la recompensa. Es probable que el hipocampo refleje su implicación almacenando y recuperando los

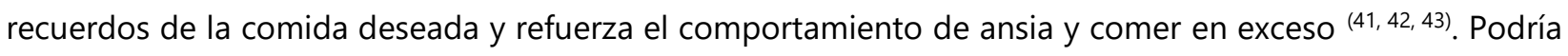
existir un procesamiento de recompensas vinculado a la $A C$ en cuanto a la búsqueda de comida hipercalórica, alta en grasas y en azúcares como una recompensa positiva ${ }^{(43)}$.

La SR materna, fue de las tres variables, la única que tuvo una influencia sobre la SR del hijo, la SR del hijo posiblemente tenga múltiples factores implicados, y uno de los principales es la madre. Se pudo comprobar que las madres pueden dirigir la SR de sus hijos en el consumo de alimentos ${ }^{(44)}$. En otros modelos, la probabilidad de presentar AC en el hijo esta inducida en parte por las características presentes en la madre como lo son la SR y la AC ${ }^{(15)}$. En cuanto al IMC del hijo se encontró que la SR, la AC y el IMC de la madre influyeron. En este contexto, posiblemente los patrones familiares, las contribuciones genéticas, la exposición prenatal a la alimentación hipercalórica, el modelado parental del uso y acceso en el entorno del niño de alimentos hipercalóricos y sabrosos por parte de la madre logren explicarlo de una manera más específica ${ }^{(15)}$. 


\section{Conclusiones}

En resumen, el IMC, la SR y la AC de la madre influyen sobre el IMC, la SR y la AC del hijo, de manera concreta la SR de la madre influyó en la SR del hijo, así mismo la SR y AC de la madre sobre la AC del hijo, y la SR, la AC y el IMC de la madre influyeron sobre IMC del hijo. Estas características presentes en la madre tanto el SP/OB, una alta SR y AC estarían presentes en los niños, y podrían ir aumentando conforme aumenta la edad, persistir en la edad adulta y ser trasmitidas hacia sus futuros hijos.

\section{Conflicto de intereses}

Los autores declaran que no tienen conflictos de intereses.

\section{Financiamiento}

Ninguno.

\section{Referencias bibliográficas}

1. Freitas FR, Moraes DE, Warkentin S, Mais LA, Ivers JF, Taddei JA. Maternal restrictive feeding practices for child weight control and associated characteristics. J Pediatr [Internet]. 2019 [consultado 15 ene 2019]; 95(2):201-08. Disponible en: https://www.sciencedirect.com/science/article/pii/S0021755717305004

2. Sahoo K, Sahoo B, Choudhury AK, Sofi NY, Kumar R, Bhadoria AS. Childhood obesity: causes and consequences. J Fam Med Prim Care [Internet]. 2015 [consultado 15 ene 2019];4(1):187-192. Disponible en: http://refhub.elsevier.com/S00217557(17)305004/sbref0150

3. Organización de las Naciones Unidas para la Alimentación y la Agricultura (FAO). Obesidad y Sobrepeso [Internet]. FAO. 2017 [consultado 17 ene 2019]. Disponible en: http://www.fao.org/about/meetings/icn2/ preparations/document-detail/es/c/253843/

4. Organización Mundial de la Salud (OMS). La obesidad entre los niños y los adolescentes se ha multiplicado por 10 en los cuatro últimos decenios [Internet]. OMS. 2017 [consultado 17 ene 2019]. Disponible en: http://www.who.int/mediacentre/ news/releases/2017/increase-childhood-obesity/es/

5. Instituto Nacional de Salud Pública (INSP). Encuesta Nacional de Salud y Nutrición: Informe final de resultados [Internet]. INSP. 2016 [consultado 19 ene 2019]. Disponible en: https://www.gob.mx/cms/uploads/attachment/file/209093/ENSANUT.pdf

6. Rodrigue C, Gearhardt AN, Bégin C. Food Addiction in Adolescents: Exploration of psychological symptoms and executive functioning difficulties in a non-clinical sample. Appetite [Internet]. 2019 
[consultado 20

oct

2019].141:104303.

Disponible

en:

https://www.sciencedirect.com/science/article/abs/pii/S0195666319300844

7. Loxton NJ. The Role of Reward Sensitivity and Impulsivity in Overeating and Food Addiction. Current Addiction Reports [Internet]. 2018 [consultado 19 ene 2019];5(2):212-222. Disponible en: https://www.ncbi.nlm.nih.gov/pubmed/27756640

8. Dietrich A, Federbusch M, Grellmann C, Villringer A, Horstmann A. Body weight status, eating behavior, sensitivity to reward/punishment, and gender: relationships and interdependencies. Frontiers in Psychology [Internet]. $2014 \quad$ [consultado 20 ene 2019];5:1073. Disponible en: https://www.ncbi.nlm.nih.gov/pubmed/25368586

9. De Decker A, Sioen I, Verbeken S, Braet C, Michels N, De Henauw S. Associations of reward sensitivity with food consumption, activity pattern, and BMI in children. Appetite [Internet]. 2015 [consultado 20 ene 2019]; 100:189-196. Disponible en: https://www.ncbi.nlm.nih.gov/pubmed/26898320

10. Falbe J, Thompson HR, Patel A, Madsen KA. Potentially addictive properties of sugar-sweetened beverages among adolescents. Appetite [Internet]. 2019 [consultado 20 oct 2019]; 133:130-137. Disponible en: https://www.sciencedirect.com/science/article/abs/pii/S0195666318301740

11.- Meule A, Gearhardt AN. Five years of the Yale Food Addiction Scale: Taking stock and moving forward. Current Addiction Reports [Internet]. 2014 [consultado 22 ene 2019];1(3):193-205. Disponible en: https://link.springer.com/article/10.1007/s40429-014-0021-z.

12. Pursey KM, Gearhardt AN, Burrows TL. The relationship between "food addiction" and visceral adiposity in young females. Physiology \& Behavior [Internet], 2016 [consultado 22 ene 2019]; 157:9-12. Disponible en: https://www.ncbi.nlm.nih.gov/pubmed/26796889

13. Loxton NJ, Tipman RJ. Reward sensitivity and food addiction in women. Appetite [Internet]. 2017 [consultado 23 ene 2019];115:28-35. Disponible en: https://pubmed.ncbi.nlm.nih.gov/27756640/

14. Maxwell AL, Loxton NJ, Hennegan JM. Exposure to food cues moderates the indirect effect of reward sensitivity and external eating via implicit eating expectancies. Appetite [Internet]. 2017 [consultado 23 ene 2019]; 111:135-141. Disponible en: https://www.ncbi.nlm.nih.gov/pubmed/28042038

15. Burrows T, Skinner J, Joyner MA, Palmieri J, Vaughan K, Gearhardt AN. Food addiction in children: Associations with obesity, parental food addiction and feeding practices. Eating behaviors [Internet]. 2017 [consultado 24 ene 2019]; 26:114-120. Disponible en: https://www.ncbi.nlm.nih.gov/pubmed/28236739

16. Clark SM, Martens K, Smith-Mason CE, Hamann A, Miller-Matero LR. Validation of the Yale Food Addiction Scale 2.0 among a bariatric surgery population. Obesity surgery [Internet]. 2019 [consultado 24 jul 2019]; 29(9):2923-2928. Disponible en: http://website60s.com/upload/files/obesity-surgery-v-29-iss-9a26.pdf.

17. Vandeweghe L, Vervoort L, Verbeken S, Moens E, Braet C. Food Aproach and Food Avoidance in Young Children: Relation with Reward Sensitivity and Punishment Sensitivity. Front Psychol [Internet]. 2016 
[consultado 25 ene 2019];7:928. Disponible en: https://pubmed.ncbi.nlm.nih.gov/27445898/. $0.3389 /$ fpsyg.2016.00928

18. Vandeweghe L, Verbeken S, Vervoort L, Moens E, Braet C. Reward sensitivity and body weight: the intervening role of food responsive behavior and external eating. Appetite [Internet], 2017 [consultado 25 ene 2019];112:150-156. Disponible en: https://www.ncbi.nlm.nih.gov/pubmed/28108344

19. Cohen J. Statistical power analysis for the behavioral sciences, (2nd ed.). Hillsdale, N.J.: Erlbaum. 1998 [consultado 25 ene 2019].

20. Carver CS, White TL. Behavioral inhibition, behavioral activation, and affective responses to impending reward and punishment: the BIS/BAS scales. J Pers Soc Psychol [Internet]. 1994 [consultado 25 ene 2019]; 67(2):319-33. Available from https://pdfs.semanticscholar.org/9b4e/eff022962192e2305bab461e70d22aa2d354.pdf

21. Jiménez LB, Acosta BR, Cuevas YM, Reséndiz PSC. Evaluación psicométrica de los sistemas de activación e inhibición del comportamiento en adultos mexicanos. Anales de Psicología/Annals of Psychology [Internet]. 2009 [consultado 25 ene 2019];25(2):358-367. Disponible en: https://revistas.um.es/analesps/article/view/88071

22. Vervoort L, De Caluwé E, Vandeweghe L, De Decker A, Wante L, Van Beveren ML, et al. Parent-Reported BIS/BAS Scales for Children: Factor Structure and Measurement Invariance Across Age and Gender. Assessment [Internet]. 2017 [consultado 25 ene 2019]; 26(7):1282-95. Disponible en: https://biblio.ugent.be/publication/8542104

23. Valdés MMI, Rodríguez MMC, Cervantes NJJ, Camarena B, De Gortari P. Traducción al español de la escala de adicción a los alimentos de Yale (Yale Food Addiction Scale) y su evaluación en una muestra de población mexicana. Análisis factorial. Salud mental [Internet]. 2016 [consultado 25 ene 2019];39(6):295-302. Disponible en: http://www.scielo.org.mx/scielo.php?script=sci_arttext\&pid=S0185-33252016000600295

24. Organización Mundial de la Salud (OMS). 10 datos sobre la obesidad [Internet]. 2009 [consultado 26 ene 2019]. Disponible en: http://www.who.int/features/factfiles/obesity/facts/es/

25. Centers for Disease Control and Prevention (CDC). Criterios de sobrepeso y obesidad en niños y adolescentes. División de Nutrición, Actividad Física y Obesidad [Internet]. 2015 [consultado 26 ene 2019]. Disponible

en: https://www.cdc.gov/healthyweight/spanish/assessing/bmi/childrens_bmi/acerca_indice_masa_corporal_ni nos_adolescentes.html

26. Secretaría de Salud (SSA). Reglamento de la Ley General de Salud en Materia de Investigación para la Salud [Internet]. 2014 [consultado 15 ene 2019]. Disponible en: http://www.diputados.gob.mx/LeyesBiblio/regley/Reg_LGS_MIS.pdf

27. Gearhardt AN, White MA, Masheb RM, Grilo CM. An examination of food addiction in a racially diverse sample of obese patients with binge eating disorder in primary care settings. Comprehensive Psychiatry [Internet]. 2013 [consultado 05 ene 2020];54(5):500-505. Disponible en: https://www.ncbi.nlm.nih.gov/pmc/articles/PMC3638060/ 
28. Pedram P, Wadden D, Amini P, Gulliver W, Randell E, Cahill F, et al. Food addiction: its prevalence and significant association with obesity in the general population. PloS one [Internet]. 2013 [consultado 05 ene 2020];8(9):e74832.

Disponible

en: https://www.ncbi.nlm.nih.gov/pmc/articles/PMC3762779/pdf/pone.0074832.pdf

29. Naghashpour M, Rouhandeh R, Karbalaipour M, Miryan M. Prevalence of food addiction among Iranian children and adolescents: Associations with sociodemographic and anthropometric indices. Medical Journal of The Islamic Republic of Iran [Internet]. 2018 [consultado 05 ene 2020];32(1):37-46. Disponible en: http://mjiri.iums.ac.ir/browse.php?a_id=4697\&slc_lang=en\&sid=1 \&printcase $=1 \& \mathrm{hbnr}=1 \& \mathrm{hmb}=1$

30. Davis C, Strachan S, Berkson M. Sensitivity to reward: implications for overeating and overweight. Appetite [online]. 2004 [consultado 06 ene 2020];42(2):131-138. Available from: https://www.sciencedirect.com/science/article/abs/pii/S0195666303001326

31. Franken $\mathrm{IH}$, Muris $\mathrm{P}$. Individual differences in reward sensitivity are related to food craving and relative body weight in healthy women. Appetite [Internet]. 2005 [consultado 06 ene 2020]; 45(2):198-201. Disponible en: https://www.ncbi.nlm.nih.gov/pubmed/15949869

32. Verbeken S, Braet C, Lammertyn J, Goossens L, Moens E. How is reward sensitivity related to bodyweight in children? Appetite [Internet]. 2012 [consultado 07 ene 2020];58(2):478-483. Disponible en: https://www.ncbi.nlm.nih.gov/pubmed/22138702

33. Reynolds CM, Segovia SA, Vickers MH. Experimental models of maternal obesity and neuroendocrine programming of metabolic disorders in offspring. Frontiers in endocrinology [Internet]. 2017 [consultado 08 ene 2020]; 8:245. Disponible en: https://www.ncbi.nlm.nih.gov/pubmed/28993758

34. Vucetic Z, Kimmel J, Totoki K, Hollenbeck E, Reyes TM. Maternal high-fat diet alters methylation and gene expression of dopamine and opioid-related genes. Endocrinology [Internet]. 2010 [consultado 08 ene 2020];151(10):4756-4764. Disponible en: https://www.ncbi.nlm.nih.gov/pubmed/20685869

35. Grissom NM, Lyde R, Christ L, Sasson IE, Carlin J, Vitins AP, et al. Obesity at conception programs the opioid system in the offspring brain. Neuropsychopharmacology [Internet]. 2014 [consultado 08 ene 2020]; 39(4):801. Disponible en: https://www.ncbi.nlm.nih.gov/pubmed/23924601

36. Brunault P, Ballon N, Gaillard P, Réveillère C, Courtois R. Validation of the French version of the Yale Food Addiction Scale: an examination of its factor structure, reliability, and construct validity in a nonclinical sample. The Canadian Journal of Psychiatry [Internet]. 2014 [consultado 09 ene 2020];59(5):276-284. Available from: https://www.ncbi.nlm.nih.gov/pubmed/25007281

37. Flint AJ, Gearhardt AN, Corbin WR, Brownell KD, Field AE, Rimm EB. Food-addiction scale measurement in 2 cohorts of middle-aged and older women. The American journal of clinical nutrition [Internet]. 2014 [consultado 08 ene 2020];99(3):578-586. Disponible en: https://www.ncbi.nlm.nih.gov/pmc/articles/PMC3927691/

38. Gearhardt AN, Corbin WR, Brownell KD. Preliminary validation of the Yale food addiction scale. Appetite [Internet]. 2009 [consultado 08 ene 2020];52(2):430-436. Disponible en: https://www.ncbi.nlm.nih.gov/pubmed/19121351 
39. Keser A, Yüksel A, Yesiltepe-Mutlu G, Bayhan A, Özsu E, Hatun S. A new insight into food addiction in childhood obesity. The Turkish Journal of Pediatrics [Internet]. 2015 [consultado 08 ene 2020];57(3):219. Disponible en: https://www.ncbi.nlm.nih.gov/pubmed/26701938

40. Magyar ÉE, Tenyi D, Gearhardt A, Jeges S, Abaligeti G, Toth AL, et al., Adaptation and validation of the Hungarian version of the Yale Food Addiction Scale for Children. Journal of Behavioral Addictions [online]. 2018 [consultado 08 ene 2020];7(1):181-188. Disponible en: https://www.ncbi.nlm.nih.gov/pubmed/29383940

41. Shapira NA, Lessig MC, He AG, James GA, Driscoll DJ, Liu Y. Satiety dysfunction in Prader-Willi syndrome demonstrated by fMRI. Journal of Neurology, Neurosurgery \& Psychiatry [Internet]. 2005 [consultado 09 ene 2020];76(2):260-262. Disponible en: https://pubmed.ncbi.nlm.nih.gov/15654046/

42. Smeets PA, de Graaf C, Stafleu A, Osch MJ, Nievelstein RA, Grond J. Effect of satiety on brain activation during chocolate tasting in men and women. Am J Clin Nutr [Internet]. 2006 [consultado 09 ene 2020]; 83(6):1297-1305. Disponible en: https://www.ncbi.nlm.nih.gov/pubmed/16762940

43.- Zhang $Y, M$ von Deneen $K$, Tian J, Gold M, Liu Y. Food addiction and neuroimaging. Current pharmaceutical design. Curr Pharm Des [Internet]. 2011 [consultado 09 ene 2020]; 17(12):1149-1157. Disponible en: https://www.ncbi.nlm.nih.gov/pubmed/21492080

44. Telzer EH, Ichien NT, Qu Y. Mothers know best: redirecting adolescent reward sensitivity toward safe behavior during risk taking. Social cognitive and affective neuroscience [Internet]. 2015 [consultado 09 ene 2020];10(10):1383-1391. Disponible en: https://www.ncbi.nlm.nih.gov/pmc/articles/PMC4590537/

Cómo citar este artículo: Santos-Flores JM, Cárdenas-Villarreal VM, Gutierrez-Valverde JM, Pacheco-Pérez LA, Guevara-Valtier MC. Sensibilidad a la recompensa, adicción a la comida y obesidad en madres e hijos.

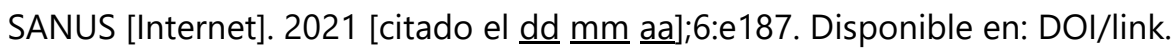

